\title{
Unexpected role of the telomere-associated protein Rap1 in protecting against mitochondrial defects and cardiac dysfunction during aging
}

\author{
Yin Cai, Michael G. Irwin, Zhengyuan Xia \\ Anaesthesiology, The University of Hong Kong, China
}

\section{Background}

With aging, the heart exhibits mitochondrial defects, leading to cardiomyocyte death, left ventricular (LV) hypertrophy and systolic/diastolic dysfunction. Repressor activator protein 1 (Rap1), an established telomere-associated protein, is essential in maintaining telomere function. Our preliminary work showed that aged Rap1 knockout mice exhibit more pronounced aging-associated phenotypes, such as earlier hair graying, massive hair loss and reduced body weight. However, the effects of Rap1 on mitochondrial function and its contribution to cardiac aging are completely unexplored. Thus, the present study investigated whether Rap1 deficiency precipitates cardiac aging and the underlying mechanism.

Methods

The cardiac structure and function of aged Rap1 wildtype and Rap1 knockout mice (85-weeks-old) were measured by echocardiography. Cardiac senescence and fibrosis were evaluated by SA- $\beta$-gal activity and Masson's Trichrome staining, respectively. The ultrastructure of mitochondria was detected by electronic microscope. Protein expressions of p53, cleaved caspase-3 (CC3), Acetyl-CoA carboxylase (ACC), phosphorylated ACC and Acyl-CoA dehydrogenase long chain (ACADL) in the heart were measured by Western blotting.

Results

Rap1 knockout aggravated aging-related cardiac structural changes and dysfunction, as evidenced by significant increases in LV posterior wall end-diastole and LV mass but reductions in ejection fraction and fractional shortening, as well as significantly impaired myocardial performance index. These changes were associated with greater cardiac senescence (increase in SA- $\beta$-gal) and fibrosis, suggesting that Rap1 deficiency precipitates cardiac aging in mice. The severe cardiac aging in the aged Rap1 knockout mice was paralleled by the greater abnormalities in the mitochondrial ultrastructure (cristae fragmentation, vacuolization), impaired fatty acid oxidation (increased ACC, phosphorylated-ACC and reduced ACADL level) and the enhanced cardiomyocytes apoptosis (increased CC3 activity), supporting that Rap1 deficiency leads to mitochondrial dysfunction and loss of cardiomyocytes during aging. Of note, p53, a driver of cell senescence and mitochondrial defects, is significantly increased in the heart of aged Rap1 knockout mice, suggesting that Rap1 deficiency may precipitate cardiac aging and mitochondrial dysfunction via enhancing p53.

Conclusions

Rap1 deficiency may cause mitochondrial defects and cardiomyocyte apoptosis via activating p53, leading to compromised cardiac structural and functional changes during aging. These findings identify a new cardiac dimension in the physiological role played by telomere-Rap1. 